\title{
SURVEILLANCE RANGE AND INTERFERENCE IMPACTS ON SELF-SEPARATION PERFORMANCE
}

\author{
Zahra Khan and Husni Idris, Engility Corporation, Billerica, MA \\ Maria Consiglio and David Wing, NASA Langley Research Center, Hampton, VA
}

\begin{abstract}
Self-separation is a concept of flight operations that aims to provide user benefits and increase airspace capacity by transferring traffic separation responsibility from ground-based controllers to the flight crew. Self-separation is enabled by cooperative airborne surveillance, such as that provided by the Automatic Dependent Surveillance-Broadcast (ADSB) system and airborne separation assistance technologies. This paper describes an assessment of the impact of ADS-B system performance on the performance of self-separation as a step towards establishing far-term ADS-B performance requirements. Specifically, the impacts of ADS-B surveillance range and interference limitations were analyzed under different traffic density levels. The analysis was performed using a batch simulation of aircraft performing self-separation assisted by NASA's Autonomous Operations Planner prototype flight-deck tool, in two-dimensional airspace. An aircraft detected conflicts within a look-ahead time of ten minutes and resolved them using strategic closed trajectories or tactical open maneuvers if the time to loss of separation was below a threshold.
\end{abstract}

While a complex interaction was observed between the impacts of surveillance range and interference, as both factors are physically coupled, self-separation performance followed expected trends. An increase in surveillance range resulted in a decrease in the number of conflict detections, an increase in the average conflict detection lead time, and an increase in the percentage of conflict resolutions that were strategic. The majority of the benefit was observed when surveillance range was increased to a value corresponding to the conflict detection look-ahead time. The benefits were attenuated at higher interference levels. Increase in traffic density resulted in a significant increase in the number of conflict detections, as expected, but had no effect on the conflict detection lead time and the percentage of conflict resolutions that were strategic. With surveillance range corresponding to ADS-B minimum operational performance standards for Class A3 equipment and without background interference, a significant portion of conflict resolutions, 97 percent, were achieved in the preferred strategic mode. The majority of conflict resolutions, 71 percent, were strategic even with very high interference (over three times that expected in 2035).

\section{Introduction}

In order to handle the expected increase in air traffic, the Next Generation Air Transportation System (NextGen) will introduce key capabilities in Air Traffic Management (ATM) [1]. Major among them is the Automatic Dependent SurveillanceBroadcast (ADS-B) system, which provides cooperative airborne surveillance enabling aircraft to exchange state and intent information with each other. ADS-B, along with other capabilities such as precise navigation and digital communication, enable shifting the ATM system towards a distributed architecture where some ATM responsibilities may be partially shouldered by aircraft systems and flight crews, thus increasing airspace capacity [2,3]. Nearterm distributed concepts include delegated separation, whereby the service provider authorizes specific aircraft to temporarily maintain separation between themselves for limited operations such as interval management. Far-term concepts include selfseparation, whereby traffic separation responsibility is transferred to the flight crew for extended periods of time. The flight crew is assisted by Airborne Separation Assistance System (ASAS) technologies to maintain separation.

ADS-B performance requirements are determined by the required performance of applications such as delegated separation and selfseparation. The current ADS-B requirements are defined in the minimum operational performance standards (MOPS) document [4], which considered near-term ADS-B applications. However, the limiting case for ADS-B performance may be far-term 
applications such as self-separation, which must meet performance levels that are required to achieve the ATM safety and resource management goals. It must be determined whether the performance of selfseparation operations is stable and predictable under a variety of conditions including variations in surveillance system performance. As a step towards establishing far-term ADS-B performance requirements, a thorough understanding of the impacts of surveillance system performance on selfseparation is needed. In particular, various aspects of the system for exchanging surveillance data may impact the self-separation performance. These aspects include: the range over which surveillance data is exchanged, the interference/noise environment, the surveillance message content and update rate, and the use of complementary systems such as the ground-based Traffic Information System-Broadcast (TIS-B) which broadcasts surveillance information about aircraft not equipped for ADS-B. Additionally, the handling of incomplete surveillance data by the ASAS and external factors such as weather and traffic density may also impact self-separation performance.

This study investigated the impact of three factors on self-separation performance: the range over which surveillance data is exchanged, referred to as the surveillance range; the interference environment, and traffic density. This study was conducted as a batch simulation using the Airspace and Traffic Operations Simulation (ATOS) hosted in the Air Traffic Operations Laboratory (ATOL) at the NASA Langley Research Center. The system for exchanging surveillance data was modeled to represent the $1090 \mathrm{MHz}$ Mode S ADS-B protocol [5]. The ASAS used in this simulation environment was the Autonomous Operations Planner (AOP), a sophisticated NASA-developed research model of an airborne automation system built for the study of advanced distributed air-ground operational concepts [6]. Previous batch studies using AOP have focused on analyzing the impacts of increasing traffic density, pilot response time, and wind uncertainty on the safety performance of self-separation under perfect surveillance system performance $[7,8,9]$. This study aims to complement these studies by gaining an understanding of the impact of key aspects of surveillance system performance on self-separation performance using the AOP model.
This paper begins by providing background on the surveillance system limitations being studied. Next, the simulation environment is described, including modeling of the AOP and the ADS-B systems. These are followed by the experiment approach including the experiment factors, scenario, and metrics used for evaluation. The results of the simulations are then presented, quantifying the impacts of surveillance range and interference on self-separation performance under the conditions of the experiment. Finally, conclusions and recommendations for future research are presented.

\section{Surveillance System Limitations}

This study investigated the impact on selfseparation performance of two main aspects of the system for exchanging surveillance data: surveillance range and interference environment.

The surveillance range refers to the distance over which the transmitted surveillance message may travel and remain strong enough to be received and interpreted. The range impacts the lead time for conflict detection. A short range may result in the self-separation algorithm of a receiving aircraft obtaining state and intent information about a transmitting aircraft on an intercept trajectory with a short lead time. A short lead time reduces the time available to perform successful or effective conflict resolution as well as the flexibility available for conflict resolution in terms of the number of available solutions. Consequently, conflict resolution may fail, increasing the possibility of loss of separation. Or it may succeed, but in the form of a less preferred tactical solution. The range of a system for exchanging surveillance data is dependent on the system hardware, most significantly the transceiver characteristics (such as transmit power and receiver sensitivity), as well as the transmission medium, e.g., atmospheric attenuation increases with inclement weather.

If the broadcast frequency (1090 $\mathrm{MHz}$ for ADSB) is heavily populated with transmissions, interference can occur and degrade the surveillance performance of the system by reducing its ability to correctly decode messages and thus, alter the reception probability of messages. The level of interference relates to the number of messages arriving at the receiving aircraft at the same time, which depends on several factors including the 
number of transmitting entities surrounding the aircraft and the message transmission rate. The transmitting entities include surrounding aircraft, transmitting ADS-B and Traffic Alert and Collision Avoidance System (TCAS) messages, and other entities such as ground-based Secondary Surveillance Radar systems which also operate on the $1090 \mathrm{MHz}$ frequency. In addition, the number of transmitting entities contributing to interference is a function of surveillance system range, since more aircraft are discovered with increased range.

\section{Simulation Environment}

This study was conducted as a batch simulation using the ATOS simulation environment hosted in ATOL at the NASA Langley Research Center. Each individual aircraft in ATOS includes its associated flight management computer, ASAS prototype, and pilot model, and is hosted on a separate computer. The resulting system is a fully-scalable, agent-based simulation of self-separation, with each individual aircraft acting independently based on data made available over simulated communication links. The following subsections describe the models for the ASAS, the communication links, and the surveillance information shared between aircraft.

\section{ASAS Modeling}

The ASAS used in this simulation was the AOP, a sophisticated NASA-developed research model of an airborne automation system built for the study of advanced distributed air-ground operational concepts. AOP provides an integrated suite of capabilities for managing trajectory changes from the flight deck perspective, including conflict detection, resolution, prevention, and trajectory constraint conformance [6]. The conflict detection (CD) function of AOP uses state and trajectory intent data received from other traffic aircraft over communication links using the ADS-B protocol in combination with ownship state data, autoflight mode settings, and flight plan information to deterministically predict future losses of separation. In the case of dropped ADS-B messages, AOP uses the previous intent data until a complete set of new data is received. The lateral minimum separation requirement was conservatively set to 5.2 nautical miles and the CD time horizon was set to ten minutes. Uncertainty buffers were applied to individual flight segments to account for trajectory prediction errors. When resolving conflicts, AOP attempts to find resolutions that are free of conflict for the next 20 minutes. For conflict resolution (CR), AOP contains both strategic and tactical capabilities. Tactical CR refers to open-loop vectors or altitude changes to resolve conflicts with no predetermined plan to reconnect to the original flight plan. Strategic CR refers to the single action of modifying the flight plan such that the conflict is resolved and the aircraft reconnects to the original flight plan. Strategic and tactical CR can use both lateral and vertical degrees of freedom. In this two-dimensional (2D) study, they were both restricted to lateral resolutions only.

AOP's strategic CR takes into account all known trajectory constraints, including trajectories of nearby traffic, airspace hazards, ownship performance limits, and required time of arrival (RTA) constraints. A genetic algorithm is employed to search within a set of pre-defined geometric patterns to generate CR trajectories that simultaneously accommodate these constraints [10]. Predefined patterns for strategic CR include path stretches with one turn out and one turn back maneuver; offsets with a turn out maneuver, a segment parallel to the original trajectory and a turn back maneuver; and direct-intercepts, with a shortcutting segment that intercepts a downstream segment. Additionally, the strategic CR algorithm can be set to use different optimization schemes including minimizing fuel burn and minimizing path deviation. For this study, the optimization scheme was set to minimize fuel burn.

AOP computes either a strategic or tactical CR based on the predicted time until the first loss of separation (LOS) and on priority rules that dictate which aircraft should give way. The priority of an aircraft is based roughly on Visual Flight Rules, e.g., the aircraft approaching from the right has priority. The burdened aircraft attempts a strategic solution unless the time remaining to LOS is five minutes or less, when it switches to tactical CR. The priority aircraft starts attempting a strategic solution if the conflict is within seven minutes and not yet resolved by the burdened aircraft. It then attempts a tactical solution if the conflict is not resolved by the burdened aircraft and the conflict is within four minutes. Once a suitable CR trajectory is calculated, and it is verified conflict free for 20 minutes based on available traffic data, it is presented to the pilot model for review and execution. Immediately upon 
execution, the aircraft's ADS-B module broadcasts the new ownship trajectory intent data.

\section{Surveillance Information Shared Between Aircraft}

A number of surveillance messages are shared between aircraft for the purposes of self-separation, as shown in Table 1. These include broadcasting of both state and trajectory-intent information for use by AOP in trajectory prediction.

Table 1 . ADS-B Report Characteristics

\begin{tabular}{|c|c|c|}
\hline $\begin{array}{l}\text { Report } \\
\text { Type }\end{array}$ & $\begin{array}{l}\text { Update } \\
\text { Rate }\end{array}$ & Content \\
\hline $\begin{array}{l}\text { Mode } \\
\text { Status }\end{array}$ & $\begin{array}{l}2-5 \\
\text { sec }\end{array}$ & $\begin{array}{l}\text { Aircraft ID, aircraft } \\
\text { capabilities, operations } \\
\text { mode, state vector quality }\end{array}$ \\
\hline $\begin{array}{l}\text { State } \\
\text { Vector }\end{array}$ & $1 \mathrm{sec}$ & Current state information \\
\hline $\begin{array}{l}\text { Target } \\
\text { State }\end{array}$ & $\begin{array}{l}1-2 \\
\text { sec }\end{array}$ & $\begin{array}{l}\text { Limited intent for tactical } \\
\text { separation }\end{array}$ \\
\hline $\begin{array}{l}\text { Trajectory } \\
\text { Change }\end{array}$ & $\begin{array}{l}1-3 \\
\text { sec }\end{array}$ & $\begin{array}{ll}\text { Trajectory intent for } \\
\text { trajectory-based } \\
\text { separation }\end{array}$ \\
\hline
\end{tabular}

For this study, aircraft intent was shared with other entities through Target State reports, which represent short-term tactical intent, and Trajectory Change (TC) reports, which represent long-term strategic trajectory-based intent. TC reports provide descriptive information of upcoming trajectory change points (TCPs) that collectively describe the next several segments of the planned trajectory. In this study, TC reports consist of a set of TCPs that were broadcast sequentially at a rate of one Hz. This approach was taken due to bandwidth limitations in the $1090 \mathrm{MHz}$ frequency such that the ADS-B 1090 $\mathrm{MHz}$ Mode S Extended Squitter message used for exchanging surveillance information can only support a single TCP. In this study, the number of TCPs was effectively unlimited by sending as many as needed (up to 12) to cover 20 minutes of intent. Thus, if four TCPs are necessary to define the next 20 minutes of the trajectory, then it would take four seconds to transmit the complete set of TCPs. Currently, the self-separation algorithm of AOP waits to receive a complete set of new TCPs before updating an aircraft's trajectory in its calculations. Message dropping due to surveillance system limitations (as discussed in the next section) might delay the reception of the complete set. For example, say a set of five TCPs is broadcast by the sending aircraft, and TCP number three in the sequence is not received due to interference. The receiving system then waits until a rebroadcast of the dropped TCP is received in a future round of TC reports before using the full set of five TCPs. Since TCPs are broadcast at $1 \mathrm{~Hz}$, it will take, at least, an additional three seconds beyond the first five-TCP broadcast to receive the missing TCP. Therefore, the receipt of the trajectory change by AOP will be delayed three seconds, which in turn delays the updating of an aircraft's trajectory by AOP and performing of CD. This can delay the detection and, consequently, resolution of a conflict, particularly if the dropping of TCPs occurs repeatedly. One purpose of this study was to assess the severity of these effects.

\section{ADS-B System Modeling}

The ADS-B model simulates real world characteristics of exchanging surveillance data by applying a message reception probability at "each receiving aircraft ... by filtering and modifying the received data stream” [5]. A successfully received message arrives at the receiving aircraft with adequate signal strength, which is related to the range of the surveillance system, and is successfully differentiated from interfering messages and decoded. As mentioned in the previous section, several different types of messages were shared between aircraft in this study. State vectors or target states can be shared using a single message while TC reports composed of several TCPs are shared using a series of messages with a single message containing a single TCP.

For the ADS-B model, "successful reception probability was developed based on the number of ground interrogators, transmitter/receiver power budget, range effects and signal overlap effects" [5]. The probability equation adds the probability of successful message reception with no interference with probabilities for successful reception in the presence of interference from various types of messages (e.g., Mode S and Mode A/C). The probability of successful message reception with no interference is the probability of no interference combined with adequate signal strength of the received message which depends on range. The 
probability terms for successful reception in the presence of interference are dependent on both surveillance range and the interference environment. A detailed model is described in [5].

The range of the system for communicating surveillance data is modeled by the system's hardware properties and the transmission medium characteristics which affect the signal strength at the receiving aircraft, as shown in the following equation $\mathrm{S}=\mathrm{P}_{\mathrm{T}} \times \mathrm{G}_{\mathrm{T}} \times \mathrm{L}_{\mathrm{T}} \times \mathrm{G}_{\mathrm{R}} \times \mathrm{L}_{\mathrm{R}} \times \mathrm{L}_{\mathrm{r}}$, which was expressed in [5] logarithmically as:

$$
\mathrm{S}=\mathrm{P}_{\mathrm{T}}+\mathrm{G}_{\mathrm{T}}+\mathrm{L}_{\mathrm{T}}+\mathrm{G}_{\mathrm{R}}+\mathrm{L}_{\mathrm{R}}+\mathrm{L}_{\mathrm{r}}
$$

where $S$ is the received power of the signal. $P_{T}, G_{T}$, $\mathrm{L}_{\mathrm{T}}, \mathrm{G}_{\mathrm{R}}$ and $\mathrm{L}_{\mathrm{R}}$ are hardware characteristics, respectively the transmitter power, the transmitter antenna gain, the transmitter cable loss, the receiver antenna gain and the receiver cable loss. $L_{r}$ is a transmission medium characteristic: the rangedependent free-space path loss which relates range to the received signal power and ultimately, to the probability of successful message reception. $\mathrm{L}_{\mathrm{r}}$ is affected, among other things, by atmospheric attenuation and weather [5]. For this study, weather effects on the surveillance system performance were not modeled. Instead, the effect of range was studied by modifying the hardware characteristics within the ADS-B model. Since Equation (1) is logarithmic, the signal power and therefore, the probability of successful message reception is related to range between the sending and receiving aircraft with an exponential decay function as shown in Fig. 1. The surveillance range is defined as the maximum distance over which a message can be sent and received successfully. With increase in the surveillance range of an aircraft, the decay function relating range to successful reception probability changes such that an aircraft not only receives messages from traffic aircraft further away but the probability of receiving messages from closer aircraft also increases.

If an aircraft is within range, a message sent from it arrives at the receiving aircraft with adequate signal strength; however, it may still be dropped due to the presence of interference which degrades the surveillance system's ability to differentiate the correct message. The interference level relates to the number of messages arriving at the receiving aircraft at the same time, which depends on several factors including the number of transmitting entities surrounding the aircraft and the message transmission rate. For this study, the aircraft that are considered as contributing to interference for a particular message are those within a circle of radius: square-root of two times the distance between the sending and receiving aircraft. This "noise" radius encompasses traffic aircraft messages whose signal strength is within three $\mathrm{dB}$ of the message under consideration and thus, significant enough to cause interference. If there is an increase in the range of a surveillance system, it is able to see messages arriving from aircraft further away. However, these messages then also experience greater interference as the "noise" radius increases.

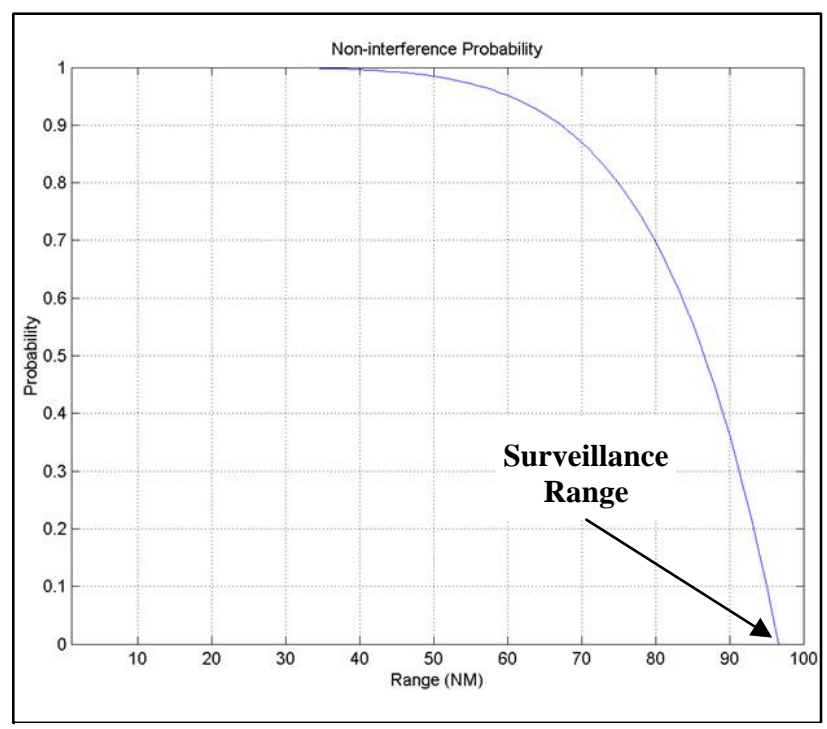

Figure 1. Relationship of Range to Probability of Successful Message Reception (figure taken from reference [5])

Given the interdependence of surveillance range and interference as described above, it is difficult to study their impact independently. For this study, the impact of interference was isolated by introducing traffic into the simulation that did not physically interact with the other aircraft. Thus, these aircraft acted only as message transmitting entities in order to vary the number of messages causing interference (representing, for example, ground radar systems) without causing conflicts. This traffic is referred to as "interference-only traffic" throughout this paper, and interference caused by messages transmitted by these aircraft is referred to as "background interference". Traffic aircraft that were simulated as actually flying and physically interacting with other aircraft are referred to as "simulated traffic aircraft" throughout this paper. 
Separately, a model option was used to simulate an ideal case of unlimited surveillance range and no interference. This option models ADS-B message reception using a step function: messages arriving from aircraft within the specified range have a message reception probability of one, and those arriving from aircraft outside the specified range have a zero reception probability. No interference effects are included in this model. To model an unlimited surveillance range, a value much larger than the maximum distance between aircraft in the simulation was used.

\section{Experiment Approach}

The experiment was conducted as a batch simulation study using a pilot model in place of human pilots. The subsections below describe the experiment scenario, factor levels and metrics.

\section{Experiment Scenario}

The experiment scenario, shown in Fig. 2, consisted of two concentric circles of 80 and $160 \mathrm{~nm}$ radii. The region between the circles served as an initialization region and the region within the inner circle as the test region. Aircraft were created at the outer boundary of the initialization region and their paths traversed the test region where experiment metrics were collected. AOP only resolved conflicts that were predicted to occur, at least partially, inside the test region. Ignoring conflicts in the initialization region served to exclude conflicts that were detected at initialization with an unrealistically short lead time, i.e., within the CD look-ahead horizon of ten minutes, thus not providing AOP adequate time to resolve them with preferred resolution approaches. Therefore, the relative dimensions of the two circles were chosen to allow approximately ten minutes flying time between them and twenty minutes of flying time in the test region, assuming an average aircraft speed of eight nm per minute. The intended result was to simulate aircraft already en-route with no conflicts at initialization entering an airspace region (the $160 \mathrm{~nm}$ diameter "test region") populated with other aircraft. Using AOP, the aircraft adjusted their trajectories as necessary to ensure they did not come closer than the lateral separation requirement of five $\mathrm{nm}$. As a conservative measure, the conflict detection and resolution criteria in AOP were set to $5.2 \mathrm{~nm}$.
The initial route of an aircraft was defined by three fixes. The first fix was randomly placed on the perimeter of the outer circle. The second fix was located randomly on the opposite side of the test region on the inner circle to ensure that the nominal route connecting the first and second fixes intersected the test region. Because AOP normally computes trajectories that may descend toward the destination, a third fix was created sufficiently far along the nominal route to avoid descent predictions within the test region, thus creating a $2 \mathrm{D}$ scenario with all aircraft in cruise. A 2D scenario design simplifies the modeling and analysis but is also considered a more challenging traffic management problem in that only lateral resolutions are available to solve conflicts.

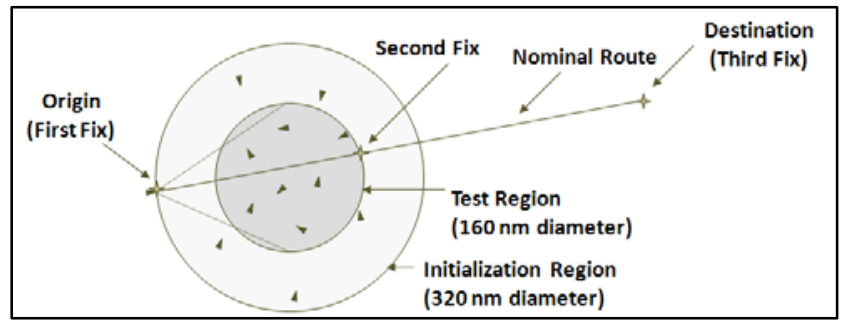

Figure 2. Experiment Scenario

A random airspeed between Mach 0.77 and 0.83 was assigned to the aircraft. All trajectories were placed at the same altitude, and only lateral resolutions were exercised. In order to maintain the desired traffic densities, each aircraft was terminated as soon as it exited the test region and another aircraft reinserted on a new route. Note that some aircraft may not have traversed the test region and were terminated upon exiting the initialization region. This happens when a conflict detected in the test region is resolved while the aircraft is still in the initialization region and the modified lateral path keeps the aircraft outside the test region. All aircraft were equipped for self-separation, and there were no wind, weather hazards or airspace restrictions.

\section{Experiment Factors}

This study investigated the impact of three factors on self-separation performance: two aspects related to exchanging surveillance data - surveillance range and background interference - and the external parameter of traffic density.

The surveillance range was varied by setting the transmitter power and keeping the other hardware 
parameters in Equation (1) constant at the Class A3 MOPS requirements for $1090 \mathrm{MHz}$ ADS-B [5]. Changing the transmitter power changed the message signal power level at the receiving aircraft. The full list of received signal power levels studied is shown in Table 2 along with the corresponding estimated surveillance range values. Since the ADS-B model directly uses transmitter power in calculating message reception probability, the value of surveillance range achieved in the simulation might be slightly different due to numerical approximation. Setting the transmitter power to the Class A3 MOPS requirements for $1090 \mathrm{MHz}$ ADS-B corresponds to a range of approximately $97 \mathrm{~nm}$ [5].

Table 2. Relationship of Surveillance Range to Received Power Level

\begin{tabular}{|l|l|l|}
\hline $\begin{array}{l}\text { Surveillance } \\
\text { Range (nm) }\end{array}$ & $\begin{array}{l}\text { Received } \\
\text { Power Level } \\
\text { (dB) }\end{array}$ & $\begin{array}{l}\text { Received Power } \\
\text { Level Compared } \\
\text { to Baseline }\end{array}$ \\
\hline 71 & 48.3 & $0.54 \mathrm{X}$ \\
\hline 86 & 50 & $0.79 \mathrm{X}$ \\
\hline 97 & 51 & Baseline: $1 \mathrm{X}$ \\
\hline 160 & 55.4 & $2.75 \mathrm{X}$ \\
\hline 200 & 57.3 & $4.27 \mathrm{X}$ \\
\hline 243 & 59 & $6.31 \mathrm{X}$ \\
\hline 324 & 61.5 & $11.22 \mathrm{X}$ \\
\hline
\end{tabular}

Table 3 shows the combinations of surveillance range and background interference levels used in the experiment. In addition to a no background interference level, two other levels, high and very high, were used. The background interference levels were chosen to be theoretically high in order to stress the self-separation capability. Pre-experiment investigation showed that high levels of background interference were needed to invoke self-separation performance that is sufficiently different from the nointerference case to be able to characterize the impacts of interference. This is partially due to the conservative settings of AOP parameters such as the CD look-ahead horizon (ten minutes) and the tactical CR override horizon (five minutes for priority aircraft and four minutes for burdened aircraft). The background interference level was varied by changing the number of "interference-only" aircraft (i.e., broadcasting information but not flying). As discussed in the previous section, this 2D study simulated aircraft self-separation at only a single flight level. However, aircraft at a particular flight level can experience interference from aircraft at other flight levels. The high and very high background interference levels in this study were caused, respectively, by approximately 965 and 1687 interference-only aircraft in the $320 \mathrm{~nm}$ diameter airspace of the scenario. These traffic levels correspond approximately to 2.0 to 3.6 times the number of traffic aircraft contributing to interference for the year 2035 as predicted by the FAA's report on $1090 \mathrm{MHz}$ Spectrum Congestion [11] using a yearly traffic growth rate of $1.7 \%$ (2009 estimate) from 2007 and representing aircraft at all flight levels in a high density Northeast corridor. The report also presented interference predictions using a higher traffic growth rate of $4.1 \%$ from 2007, based on an earlier (2008) traffic prediction. Compared to the these higher interference predictions, the background interference traffic levels used in this study correspond approximately to one to two times the number of traffic aircraft contributing to interference in the year 2035. The interference-only aircraft may also represent interference sources such as radar interrogations and TIS-B that use the $1090 \mathrm{MHz}$ channel.

\section{Table 3. Experiment Matrix for All Traffic Density Levels}

\begin{tabular}{|l|l|l|l|l|l|l|l|}
\hline $\begin{array}{l}\text { Background } \\
\text { Interference } \\
\text { Level }\end{array}$ & \multicolumn{7}{|l|}{ Surveillance Range (nm) } \\
\hline & 71 & 86 & 97 & 160 & 200 & 243 & 324 \\
\hline None & X & X & X & X & & X & X \\
\hline High & X & & X & X & X & X & X \\
\hline Very High & X & & X & X & X & X & X \\
\hline $\begin{array}{l}\text { Special case: unlimited surveillance range and no } \\
\text { interference }\end{array}$ \\
\hline
\end{tabular}

As an ideal baseline, a case with unlimited surveillance range and no interference was also run as described in ADS-B System Modeling section. As Table 3 shows, more low surveillance range values were simulated for the no background interference case whereas, for the high and very high interference cases, more high surveillance range values were simulated. This decision was based on preexperiment observations that peak self-separation performance is reached at lower surveillance ranges under the lower interference level, and hence, more 
data points may be needed at lower surveillance ranges in this case.

Finally, three different traffic levels were studied: 30, 40 and 60 aircraft in the $320 \mathrm{~nm}$ diameter airspace of the experiment scenario resulting in traffic densities of 3.73, 4.97 and 7.46 aircraft/10000 $\mathrm{nm}^{2}$. Based on the historical traffic analysis in [7], these densities correspond respectively to 2.07X, 2.76X and $4.14 \mathrm{X}$ of the mean traffic density (1.8 aircraft/10000 $\mathrm{nm}^{2}$ ) in 2004 of a median-density en route traffic sector (ZOA31 of the Oakland center). Higher traffic densities cause more interference due to increase in the number of surveillance messages and result in a more constrained solution space for CR. The experiment matrix in Table 3 was repeated for each traffic density level.

\section{Experiment Metrics}

Surveillance system and self-separation performance metrics were used to characterize the impact of the experiment factors. Surveillance system metrics included:

- Percentage of messages received: defined as the ratio of the total number of messages received to the total number of messages transmitted for all flights in the simulation

- Percentage of in-range messages received: defined as the ratio of the total number of messages received to the total number of messages transmitted from flights that are within range of a receiving aircraft

Self-separation performance metrics included:

- Number of conflict detections

- Average conflict detection lead time: defined as the mean predicted time to the first loss of separation, sampled at first detection, for all conflict detections

- Percent strategic conflict resolution: defined as the ratio of the number of strategic conflict resolutions to the total number of strategic and tactical conflict resolutions for all aircraft

- Number of losses of separation relative to the number of conflicts detected
The total number of conflict detections was used as a basis for several metrics as defined above. In self-separation operations, a conflict may be detected by one or both aircraft involved in the conflict and may also be resolved by one or both aircraft. The total number of conflict detections may include cases where both aircraft detect the same conflict, in which case the conflict is counted twice in the total number of conflict detections. Additionally, the total conflict count is calculated through post-analysis of AOP output data. AOP performs conflict detection every ten seconds and records information about any conflicts detected. The same conflict reappears in each of these records until it is resolved or ceases to be predicted. The post-analysis then associates a time-contiguous set of records for a conflict between the same two aircraft with a single conflict. Therefore, a borderline conflict (one that is at the boundary of separation loss) is counted as multiple conflicts if it "flickers" or disappears and reappears several times. These factors may result in the number of conflict detections overestimating the number of conflicts. On the other hand, a so-called "sidewalk" situation may result in under-counting conflicts. A sidewalk situation occurs when two aircraft in conflict both maneuver to resolve the conflict in a way that causes another conflict between them, such as sometimes happens when two people simultaneously yield to each other as they approach on a sidewalk. If there is no time interruption in the AOP conflict records, the resulting conflict is counted as part of the original conflict that caused the sidewalk situation. The over- and under- counting contributed to variability in the conflict detection metric, as discussed in the Results section. Nevertheless, the number of conflict detections corresponds to the number of actionable events for the aircraft involved and, thus, is considered an appropriate performance metric for self-separation.

\section{Results}

This section presents the simulation results for the combinations of the experiment factors presented in Table 3 demonstrating their impact on the metrics presented in the previous section. In some cases, trend lines were added to the data using Matlab to fit a power equation of the form $\left(a \times x^{n}+b\right)$. 


\section{Percentage of Messages Received}

The impacts of surveillance range and interference on the percentage of messages received are presented first, as they explain most of the impacts on self-separation performance presented in the following sections. The percentage of messages received was measured over all messages transmitted from all aircraft in the simulation, both within and outside the surveillance range of a receiving aircraft to determine the combined effects of range and interference. In Fig. 3, the percentage of messages received is plotted as a function of surveillance range for each background interference level. The data for the 30-, 40-, and 60-aircraft traffic density cases were essentially coincident; the figure shows data for the 30-aircraft case. (The impact of traffic density on self-separation performance is discussed in the following sections).

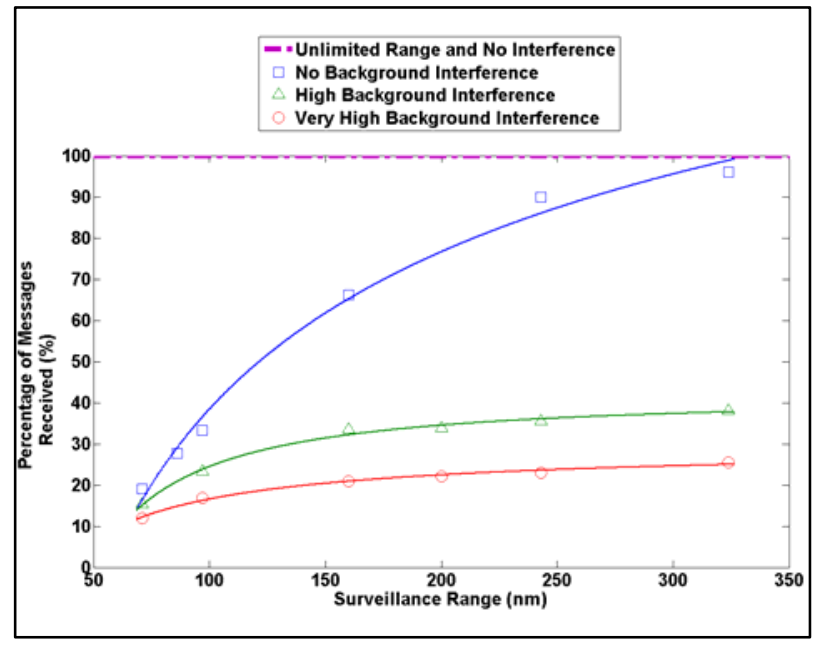

Figure 3. Effect of Surveillance Range and Background Interference on Percentage of Messages Received for 30 Aircraft Case

Under unlimited surveillance range and no background interference, all of the messages transmitted were received as expected (shown in the figure as a horizontal dashed line because the range value used for this case is off the chart). With no background interference (the blue curve), the effect of surveillance range was the most significant. At low surveillance range, few messages were received because most of the messages were transmitted from aircraft outside the range of a receiving aircraft. As the surveillance range was increased, the percentage of messages received also increased, reaching almost 100 percent at $324 \mathrm{~nm}$ range, as expected given the scenario's similarly sized diameter. The improvement was realized because of increased message transmission power (hence, reduced decay), which increased range allowing more aircraft to fall within range and increased the reception probability of messages transmitted from aircraft already within range. Even without background interference, interference effects from the simulated traffic aircraft also increased with increasing the surveillance range. Hence, the marginal increase in message reception as the surveillance range was increased diminished. With high and very high background interference, message reception also increased with surveillance range, although at significantly suppressed levels, particularly at high range values where more interference-only aircraft were detected.

To further investigate the effect of interference on message reception, the percentage of messages received from the messages transmitted only from within the surveillance range of the receiving aircraft is plotted in Fig. 4 at the three background interference levels for the 30 aircraft case. With no background interference, the percentage of in-range messages received (Fig. 4) was higher than the percentage of messages received measured over all messages (Fig. 3). Message reception increased with increasing surveillance range because the higher signal power reduces the probability of message dropping. Overall, interference effects diminished the beneficial effect of increasing surveillance range and tended to negate them at high and very high

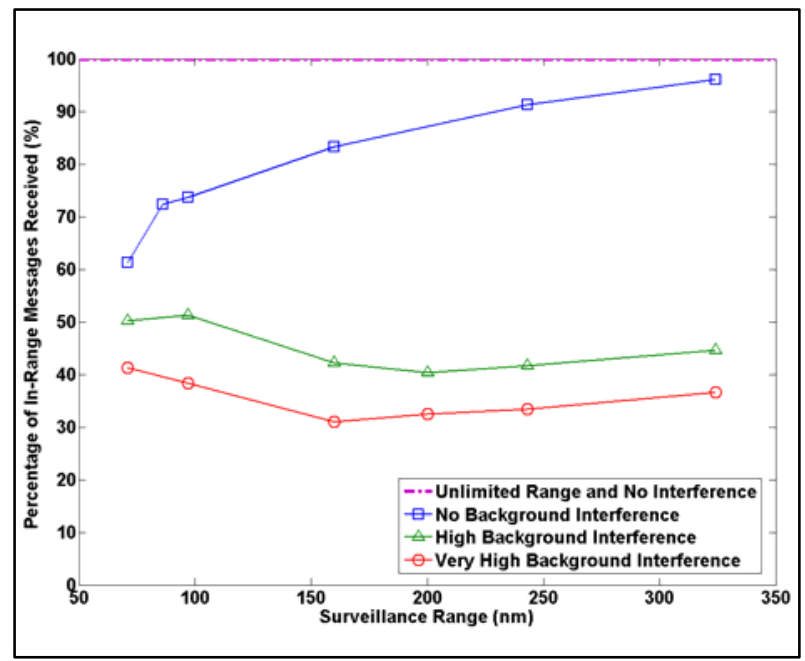

Figure 4. Effect of Surveillance Range and Background Interference on Percentage of InRange Messages Received for 30 Aircraft Case 
background interference levels. The curves for the high and very high background interference levels showed an unexpected initial negative trend up to ranges of 150-200 nm, where the interference effects tended to outweigh the positive effects of increasing surveillance range, followed by a small positive trend at higher ranges, indicating a complex interaction between the range and interference effects.

A possible explanation for the interaction follows. Increases in range add signal power, and hence improve the reception probability of all the messages transmitted including those transmitted from simulated traffic aircraft at distances much smaller than the range. Increases in range also result in additional messages that are transmitted from within range as more simulated traffic aircraft are within range. The added messages exhibit more decay and interference effects, because they are transmitted over longer distances, and therefore, their reception probability is relatively low. However, the additional messages constitute a fraction of the total messages received, that becomes increasingly smaller with further increase in range. Hence, the contribution of the additional messages to reducing the reception rate diminishes at high range values allowing, eventually, a small positive trend with range to be observed due to the positive impact of increased signal power due to range.

\section{Number of Conflict Detections}

Fig. 5 shows the impact of surveillance range and background interference on the number of

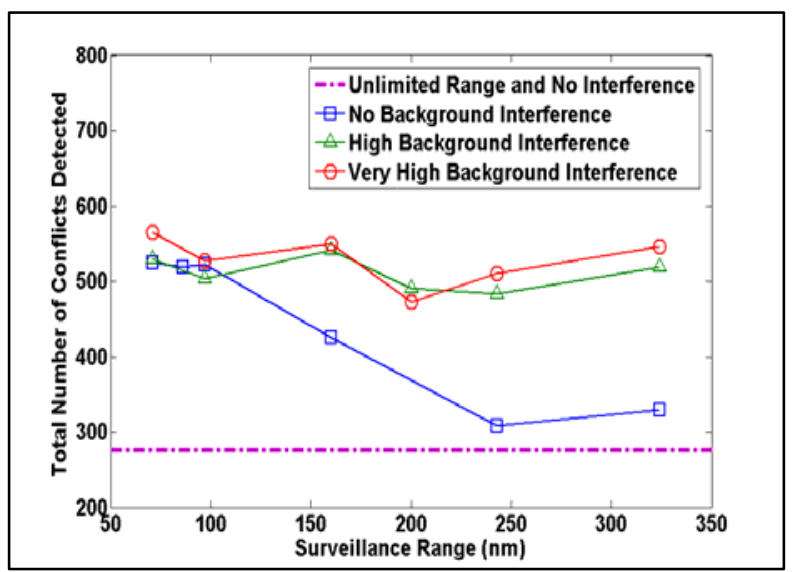

Figure 5. Number of Conflict Detections for 30 Aircraft Case (2.07X of 2004 traffic levels) conflict detections by all aircraft, for the 30 aircraft traffic density case. For the baseline condition of unlimited surveillance range and no interference, just under 300 conflicts were detected by all aircraft (shown again in the figure as a dashed horizontal line because the range value is off the chart). The effect of surveillance range was the most significant for the no background interference case (the blue curve). At surveillance range values approaching the diameter of the scenario of $320 \mathrm{~nm}$, the number of conflicts detected is close to those detected in the baseline condition. This result was expected because most aircraft within the simulation were within range and most messages from these aircraft were received, as was shown in Fig. 4.

As the surveillance range was decreased, the number of conflicts detected increased, as expected. For these cases, there was an increase in message dropping due to signal power decay and due to interference from the other aircraft messages. Message dropping can cause $\mathrm{CD}$ to give false alerts and thus, increase the number of detected conflicts, because an aircraft uses outdated, inaccurate trajectory information from another aircraft, and hence, may predict separation losses that are not real. Inaccurate traffic trajectories may also increase the number of missed alerts; missed alerts result in late detections or losses of separation (analyzed in later sections) that may or may not increase the conflict detection count. Another potential cause of the increase of conflict detections are secondary conflicts which result when an aircraft maneuvers to resolve a conflict with one aircraft, only to cause additional conflicts with the same or other aircraft, immediately or later in time. AOP's CR algorithm ensures that the conflict resolution maneuvers are free of conflict with all aircraft for a 20 minute horizon for strategic resolutions and a 12 minute horizon for tactical resolutions. However, message dropping delays the updating of traffic trajectories, and using obsolete trajectories to compute CR maneuvers may cause secondary conflicts when the trajectories are updated. Note that immediate secondary conflicts are not counted because the conflicts need to be separated in time to be counted as separate in this analysis.

At high and very high levels of background interference, the number of conflict detections generally remained elevated even as surveillance range was increased as shown in Fig. 5. At low surveillance ranges, the effect of background 
interference was limited by the small range, and hence, the number of conflict detections was similar to the no background interference case. However, the effect relative to the no background interference case was pronounced at higher surveillance range values. The number of conflict detections was essentially flat with respect to surveillance range at high and very high background interference levels. This is explained partially by the lack of constant trend in the message reception rate at high and very high background interference levels, as was shown in Fig. 4. The lack of consistency in the conflict detection count may be related to the various sources of variability in this metric discussed under Experiment Metrics.

Similar observations were made in the conflict detection counts for the 40 and 60 aircraft traffic density levels, shown respectively in Fig. 6 and Fig. 7. The trends are the same as for the 30 aircraft case. However, the number of conflict detections increased significantly with traffic density, an expected result since a higher traffic density represents a more constrained airspace. In Fig. 7, the number of conflict detections for the no background interference case drops below the baseline. Although not fully understood, one possible source of this behavior is the variability discussed in the Experiment Metrics section involving the potential for under- and overcounting conflicts, which is believed to be different between the baseline case and the no background interference case.

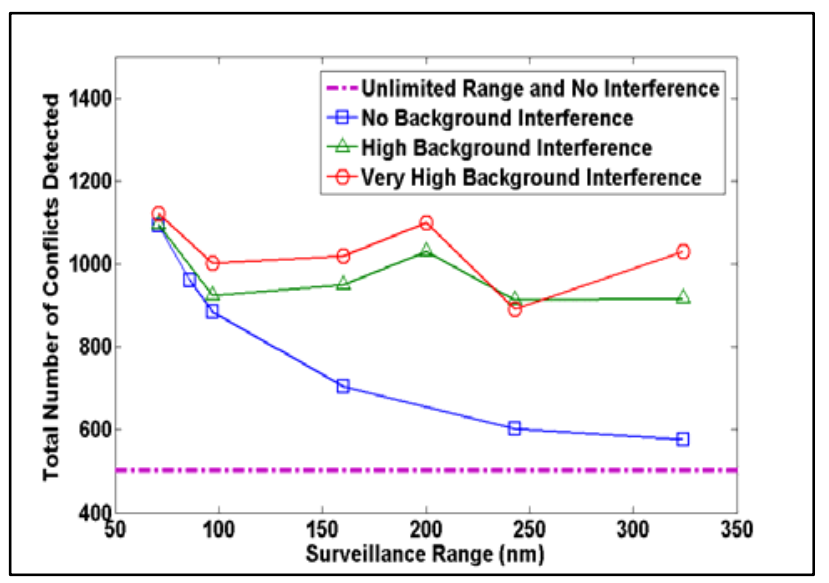

Figure 6. Number of Conflicts Detections for 40 Aircraft Case (2.76X of 2004 traffic levels)

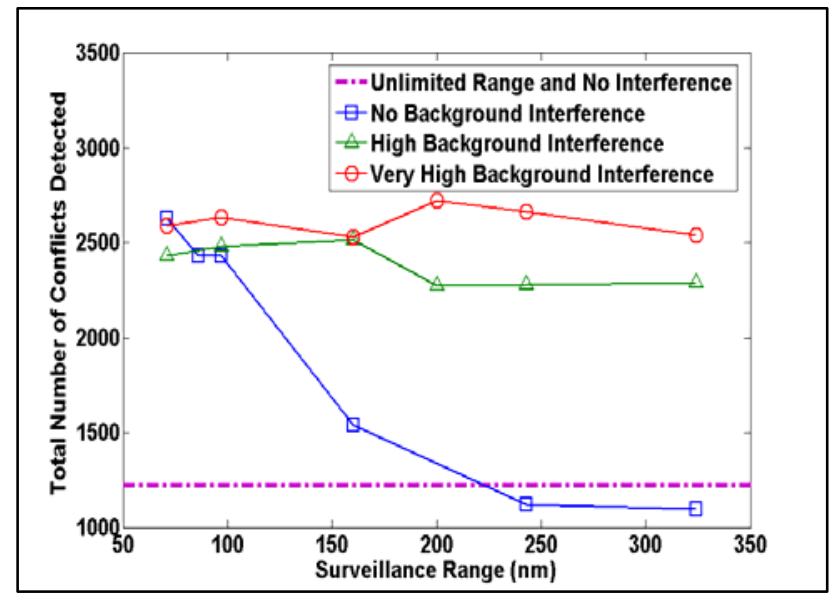

Figure 7. Number of Conflicts for 60 Aircraft Case (4.14X of 2004 traffic levels)

\section{Average Conflict Detection Lead Time}

Reduced surveillance range can cause delay in receiving trajectory information from surrounding traffic, and, therefore, a potential delay in some conflict detections. Even for aircraft within range, message dropping due to signal decay and interference impacts the availability of traffic trajectory information for timely conflict detection. The average CD lead time was used as a metric to investigate the timeliness of conflict detection with varying surveillance range and background interference. Conflict detections formed the basis for measuring the average CD lead time, and hence, the number of conflict detections represented the sample size for each experiment run, with values ranging from 300 to 2700, as shown in Figs. 5-7.

AOP was configured with a CD look-ahead horizon of ten minutes as a threshold for earliest alerting because detections at longer horizons may be unreliable due to various uncertainties in trajectory prediction. Fig. 8 presents a histogram of CD lead times for the baseline case as well as several range cases at the no background interference level. For the baseline case, the distribution of CD lead times had a peak very close to ten minutes. Any conflicts that could have been detected earlier than ten minutes were instead detected at or close to ten minutes thus causing the peak near ten minutes. For other range and interference cases, use of a CD look-ahead horizon resulted in a bi-modal distribution for the CD lead time, with one peak close to ten minutes and a second peak whose location and relative magnitude 
to the first peak vary with the surveillance range and interference combinations in the experiment matrix. As can be seen from Fig. 8, all the range values presented have a peak in the nine to ten minute time bin. The lower range cases have a second peak in the four to six minute time bins.

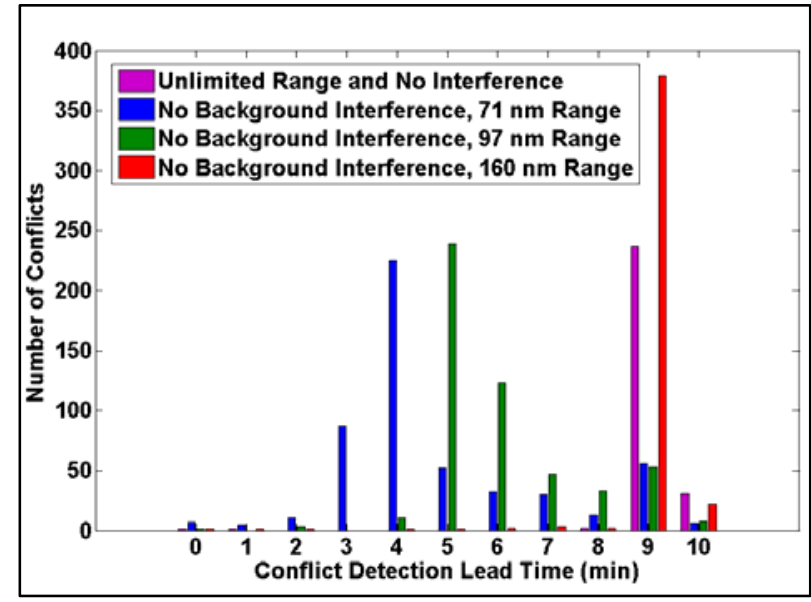

Figure 8. Distribution of Conflict Detection Lead Times for Some Range Values for No Background Interference and Baseline, 30 Aircraft Case

Fig. 9 shows the impact of surveillance range and background interference on the average CD lead time for the 30 aircraft case. For the baseline case of unlimited surveillance range and no background interference, the average $\mathrm{CD}$ lead time nearly coincided with the ten-minute CD look-ahead horizon used in the experiment. For the remaining cases, the average $\mathrm{CD}$ lead time increased with increase in surveillance range and decreased with increase in background interference, as expected. The average CD lead time data followed the same trends of the percentage of messages received, shown in Fig. 3. As the percentage of messages received increases with surveillance range, a complete set of TCPs is available sooner for use in CD. As background interference is introduced, a larger number of messages are dropped, and the availability of an accurate and complete set of TCPs for CD is delayed. The result was a significant reduction in the average CD lead time, as confirmed by a Tukey simultaneous pair wise comparison test.

Fig. 9 also shows the relationship of the average CD lead time to AOP's tactical CR override horizon, the time within which unresolved and newly detected conflicts are resolved with tactical maneuvers. In this study, the tactical CR override horizon was set conservatively to five minutes (shown as a horizontal line) for burdened aircraft and four minutes for priority aircraft. At a surveillance range of $71 \mathrm{~nm}$, the average CD lead time for all interference cases was close to or fell below the five-minute tactical CR override horizon. At higher surveillance ranges, the average $C D$ lead time was greater than the tactical override horizon; the difference between average CD lead time values and the tactical override horizon diminished at high and very high background interference levels. Thus, a greater percentage of CD lead times were below five minutes, and tactical resolutions were attempted for the corresponding conflicts. This effect is discussed in the next subsection.

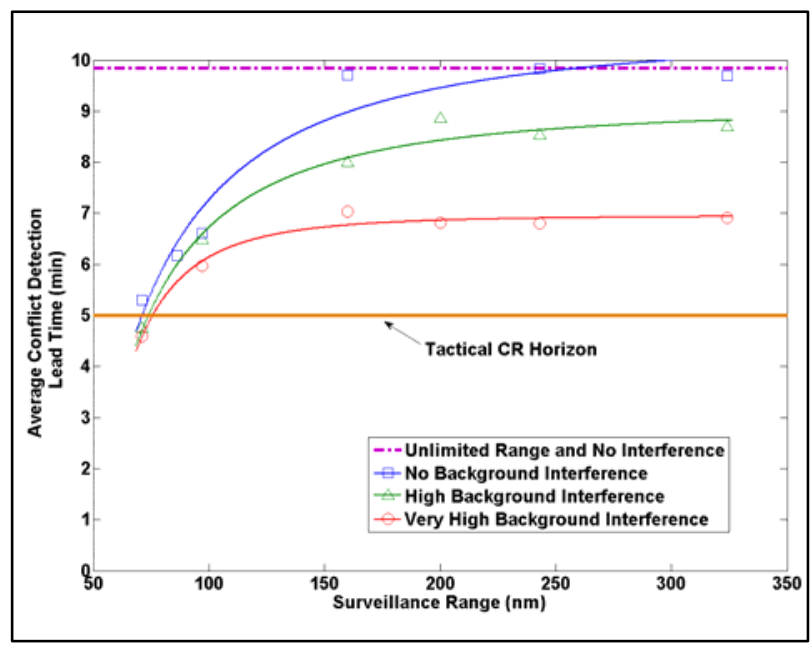

Figure 9. Effect of Surveillance Range and Background Interference on Average Conflict Detection Lead Time for 30 Aircraft Case

As Fig. 9 shows, the change in average CD lead time was pronounced at surveillance range values below about $160 \mathrm{~nm}$ and minimal beyond $160 \mathrm{~nm}$. This behavior highlights the dependence of the CD performance on the CD look-ahead horizon of the ASAS, in this case ten minutes. When two aircraft come within range of each other, the earliest conflict between them that can be detected is if they travel along a straight line toward each other, as shown in Fig. 10; this conflict scenario is the most sensitive to the magnitude of surveillance range. Since the earliest conflict that AOP detects is ten minutes away, and the average aircraft speed in the simulation was approximately $8 \mathrm{~nm}$ per minute, the earliest 


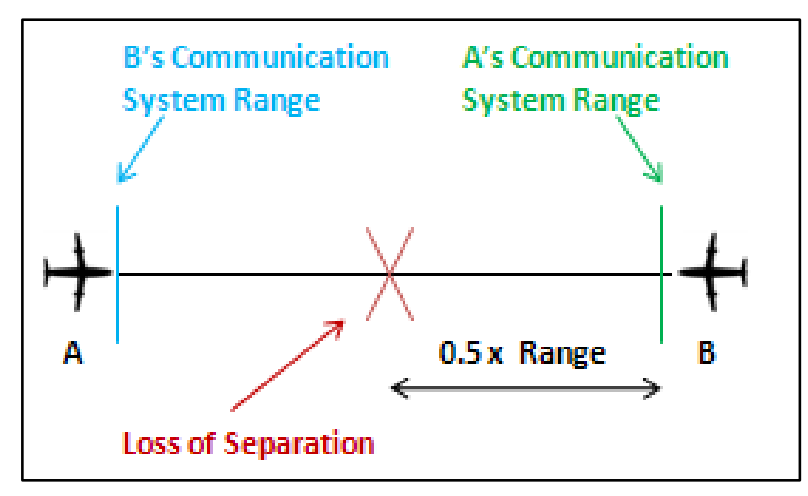

Figure 10. Worst Case Conflict Situation

conflict that can be detected is when the two aircraft are $160 \mathrm{~nm}$ apart. For example, as shown in Fig. 10, assuming both aircraft are flying the average speed the conflict would be $80 \mathrm{~nm}$ away from each aircraft. Therefore, if the surveillance range is larger than 160 $\mathrm{nm}$, a conflict could only be detected by AOP when the aircraft are $160 \mathrm{~nm}$ apart, despite coming within range and exchanging intent information earlier. Thus, increase in the surveillance range up to this value can correspondingly increase the average $C D$ lead time, as can be seen in Fig. 9. However, at higher surveillance ranges, the marginal benefit of earlier availability of intent information should have little effect because AOP does not predict conflicts earlier than ten minutes. Increasing surveillance range beyond $160 \mathrm{~nm}$ could have a small benefit, as can be seen in Fig. 9, since AOP uses intent data to ensure that trajectories are free of conflict for 20 minutes when resolving conflicts predicted in ten minutes. Additionally, increasing range beyond 160 $\mathrm{nm}$ results in the reception probability for messages transmitted from aircraft at distances less than 160 $\mathrm{nm}$ from the receiving aircraft also increasing, as discussed in the previous section, which might reduce delays in reception of intent information and thus CD.

The relationship between the average CD lead time and traffic density was analyzed for various surveillance ranges at each of the three background interference levels. Fig. 11 presents data for the minimum $(71 \mathrm{~nm})$ and maximum $(324 \mathrm{~nm})$ surveillance range values and all the background interference levels tested. The average CD lead time did not vary with traffic density for a given surveillance range and interference level. This observation was confirmed by conducting a Tukey simultaneous pair-wise comparison test at a $95 \%$ confidence level.

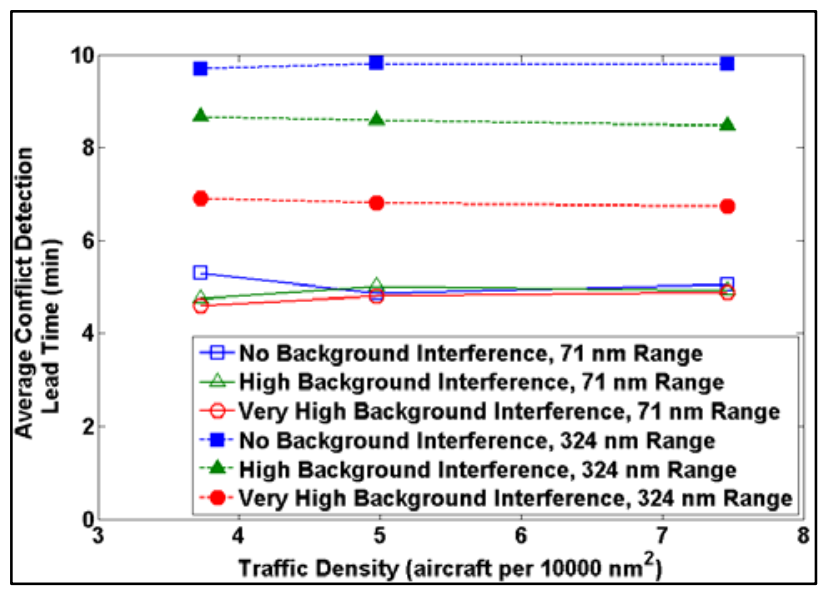

Figure 11. Effect of Traffic Density on Average

Conflict Detection Lead Time for $71 \mathrm{~nm}$ and 324 nm Surveillance Range Cases

\section{Strategic versus Tactical Conflict Resolution}

In the previous section, it was seen that early availability of traffic intent information increases CD lead times which may enable AOP to resolve more conflicts strategically rather than tactically. Fig. 12 shows the impact of surveillance range and background interference on the percentage of conflict resolutions that were strategic (rather than tactical) for the traffic density of 30 aircraft. As expected, in the baseline case of unlimited surveillance range and no interference, conflict resolutions were nearly

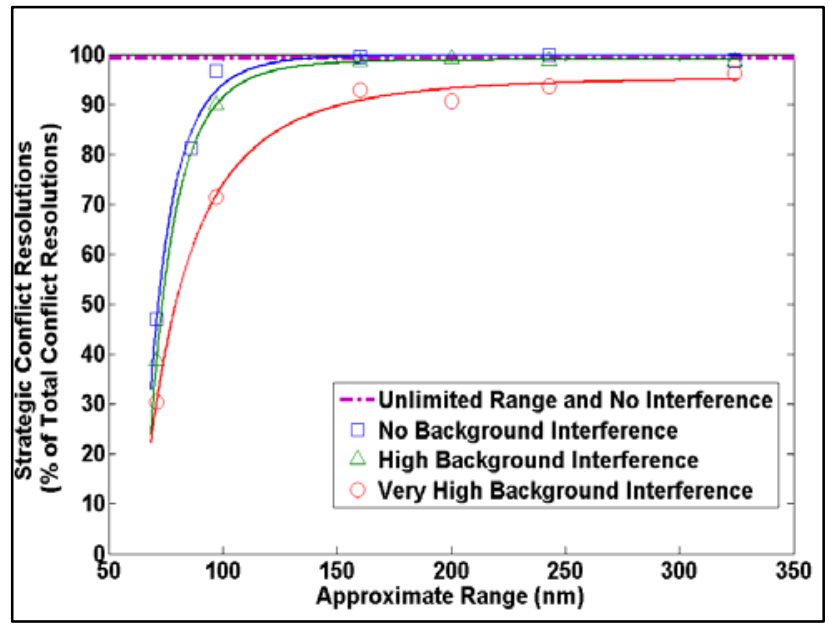

Figure 12. Effect of Surveillance Range and Background Interference on Strategic Conflict Resolutions for 30 Aircraft Case 
$100 \%$ strategic (shown in the figure as a horizontal dashed line because the range value used for this case is off the chart). For the remainder of the cases, a greater surveillance range resulted in a larger percentage of conflict resolutions that were strategic. The result was expected because higher surveillance ranges allow conflicts to be detected and traffic intent information to be available sooner, and thus more time is available to attempt a strategic CR.

Under higher background interference levels, the percentage of conflict resolutions that were strategic was lower, probably due to a larger number of messages being dropped, thus decreasing the likelihood of complete traffic intent information being available early enough to attempt strategic CR. The percentage of conflict resolutions that were strategic was approximately $97 \%$ at $97 \mathrm{~nm}$ surveillance range for the no background interference case but only approximately $71 \%$ for the very high background interference case. At a degraded surveillance range of $71 \mathrm{~nm}$, there was a significant drop in the percentage of conflict resolutions that were strategic for all background interference cases (only 20 to $40 \%$ strategic CR).

The percentage of resolutions that are strategic is related to the CD lead time and to AOP's tactical CR override horizon. For example, the average $C D$ lead time in the very high background interference case was relatively low at 6.5 minutes (see Fig. 9), which resulted in a large percentage of conflicts detected within the tactical CR override horizon. For these cases, strategic resolution was not attempted. Similarly, for all background interference cases and a surveillance range of $71 \mathrm{~nm}$, the average CD lead time was at or below the five minute tactical CR override horizon (Fig. 9). Reducing the tactical CR override horizon should result in a lower percentage of CD lead times falling below it and thus fewer conflicts requiring tactical resolution.

A correlation test confirmed that the average CD lead time (Fig. 9) and the percentage of conflict resolutions that were strategic (Fig. 12) were strongly correlated. The correlation coefficients are 0.824, 0.919 and 0.997 for the no, high, and very high background interference cases, respectively. A t-test confirmed these correlation coefficients to be significant at the 95\% confidence level with p-values of $0.0438,0.0095$ and $1.0027 \mathrm{e}-5$, respectively. Due to this correlation, the rate of change of the percentage of strategic conflict resolutions is greatly attenuated beyond $160 \mathrm{~nm}$, similarly to the average CD lead time.

The relationship between the percentage of conflict resolutions that were strategic and traffic density was analyzed at the various surveillance ranges and background interference levels. As was the case for the average CD lead time, the percentage of strategic conflict resolutions did not show a trend with traffic density.

\section{Losses of Separation}

As shown in Table 4, the total number of losses of separation (LOSs) experienced for all runs at a particular traffic density increased with increasing traffic density. Missed alerts, latency in detecting conflicts, ineffectiveness of tactical resolutions, or delays in pilot-model execution of resolutions may have contributed to these LOSs. In this batch study, few LOSs occurred relative to the total number of conflict detections. For example, for the 60 aircraft case, only 13 LOSs were reported compared to a total of 47517 conflict detections and the maximum number of LOSs in any given run was four.

Table 4. LOS Data for Various Traffic Densities

\begin{tabular}{|c|c|c|c|}
\hline $\begin{array}{l}\text { Traffic } \\
\text { Density } \\
\text { Level (\# } \\
\text { aircraft) }\end{array}$ & $\begin{array}{l}\text { Number } \\
\text { of LOSs } \\
\text { for All } \\
\text { Runs }\end{array}$ & $\begin{array}{l}\text { Number } \\
\text { of Conflict } \\
\text { Detections } \\
\text { for All } \\
\text { Runs } \\
\end{array}$ & $\begin{array}{l}\text { Ratio of } \\
\text { LOSs to } \\
\text { Conflict } \\
\text { Detections } \\
\text { for All Runs }\end{array}$ \\
\hline 30 & 0 & 9142 & 0 \\
\hline 40 & 3 & 17323 & 0.00017 \\
\hline 60 & 13 & 42427 & 0.00031 \\
\hline
\end{tabular}

Given the small numbers of LOSs, no trend was observable with respect to surveillance range or background interference level. In general, the low frequency of LOS in these scenarios indicates the high robustness of the AOP conflict detection and resolution algorithms with conflicts of random geometry and random background traffic, even in the absence of the vertical resolution degree of freedom.

\section{Conclusions}

This paper presented an analysis of the impact of ADS-B surveillance range and interference 
limitations on self-separation performance under different traffic density levels. Using a 2D scenario with no wind or weather effects, and under the modeling assumptions for the ADS-B and airborne separation assistance technologies (AOP) simulated in the experiment, a complex interaction was observed between the impact of surveillance range and interference as both factors are physically coupled. For example, under high background interference levels, the trends in the percentage of messages received from aircraft within surveillance range and the number of conflict detections were not constant over the surveillance range values. This behavior likely occurred because the resultant impact depended on balancing the opposing effects of increasing signal power and increasing interference, as surveillance range increases. Overall, as expected, an increase in surveillance range resulted in a decrease in the number of conflict detections, an increase in the average conflict detection lead time, and an increase in the percentage of conflict resolutions that were strategic, all of which are considered benefits. These benefits were attenuated at higher background interference levels. Dependence on the parameters of the separation assistance algorithms was also observed. For example, the majority of the benefit was observed when range was increased to $160 \mathrm{~nm}$ which is the average relative separation between head-on aircraft for conflicts detected at the ten-minute conflict detection lookahead time. For the MOPS Class A3 surveillance range requirement $(97 \mathrm{~nm})$, AOP was able to resolve conflicts strategically $97 \%$ of the time with no background interference and $71 \%$ of the time even with very high background interference. An increase in traffic density resulted in a significant increase in the number of conflict detections and a small increase in the number of LOSs but had no effect on the conflict detection lead time or the percentage of conflict resolutions that were strategic.

\section{Future Work}

This study focused on the impact of surveillance range and interference environment on selfseparation performance. The impacts of several other surveillance system characteristics on self-separation performance need to be studied. These include the surveillance message content and frequency and the use of complementary systems such as TIS-B in case of unavailability of information via ADS-B for unequipped aircraft. The ASAS approach for handling dropped messages should also be investigated to identify opportunities to mitigate the impact of surveillance system limitations on selfseparation performance. For example, AOP (the ASAS used in this study) requires a complete TC report set for a traffic aircraft to perform CD. In the presence of message dropping, the reception of a complete TC report set can experience delays and, thus, delay accurate CD. To mitigate this effect, the ASAS may be designed to perform CD based on partial intent information (e.g., partial TC report sets). Finally, the analysis may be performed in more realistic scenarios involving vertical $\mathrm{CR}$, wind and weather effects, and mixed operations of selfseparating aircraft and aircraft managed by a ground system.

\section{References}

[1] Joint Planning and Development Office, "Next Generation Air Transportation System Integrated Plan,” URL: http://www.jpdo.gov/library/NGATS_v1_1204r.pdf.

[2] Wing, D., 2005, “A Potentially Useful Role for Airborne Separation in 4D-Trajectory ATM Operations," Proceedings of the 5th AIAA Aviation Technology Integration and Operations (ATIO) Conference, AIAA-2005-7336.

[3] Green, S. M., K. D. Bilimoria, and M. G. Ballin, 2001, "Distributed Air/Ground Traffic Management for En Route Flight Operations.” Air Traffic Control Quarterly, Vol. 9, No. 4, pp. 259-285.

[4] RTCA, 2009, "Minimum Operational Performance Standards for $1090 \mathrm{MHz}$ Extended Squitter Automatic Dependent Surveillance Broadcast (ADS-B) and Traffic Information Services - Broadcast (TIS-B),” DO-260B.

[5] Chung, William W., and Ronald Staab, 2006, “A 1090 Extended Squitter Automatic Dependent Surveillance - Broadcast (ADS-B) Reception Model for Air-Traffic-Management Simulations”, Proceedings of AIAA Modeling and Simulation Technologies Conference and Exhibit, AIAA-20066614.

[6] Karr, D., D. Roscoe, and R. Vivona, 2006, “An Integrated Flight-Deck Decision-Support Tool in an Autonomous Flight Simulation”, Proceedings of 
AIAA Modeling and Simulation Technologies Conference and Exhibit, AIAA-2006-5261.

[7] Consiglio, Maria C., Sherwood T. Hoadley, David J. Wing, and Brian T. Baxley, 2007, "Safety Performance of Airborne Separation: Preliminary Baseline Testing", Proceedings of the 7th AIAA Aviation Technology Integration and Operations (ATIO) Conference, AIAA-2007-7739.

[8] Consiglio, Maria, Sherwood Hoadley, David Wing, Brian Baxley and Danette Allen, 2008, "Impact of Pilot Delay and Non-Responsiveness on the Safety Performance of Airborne Separation", Proceedings of the $26^{\text {th }}$ Congress of International Council of the Aeronautical Sciences (ICAS), AIAA2008-8882.

[9] Consiglio, Maria, Sherwood Hoadley, and Danette Allen, 2009, "Estimation of Separation Buffers for Wind-Prediction Error in an Airborne Separation Assistance System", Proceedings of the Eighth USA/Europe Air Traffic Management Research and Development Seminar.

[10] Vivona, R. A., D. A. Karr, and D. A. Roscoe, 2006, "Pattern-Based Genetic Algorithm for Airborne Conflict Resolution”, Proceedings of the AIAA Guidance, Navigation and Control Conference and Exhibit, AIAA-2006-6060.

[11] $1090 \mathrm{MHz}$ Spectrum Analysis Team Surveillance and Broadcast Services Program, 2009, "Final Report on 1090 MHz Spectrum Congestion in 2035 and Performance of Systems Operating in the Band”, Version 1.0, Federal Aviation Administration.

\section{Acknowledgements}

Software development support for this work was provided by Tarek El-Wakil from the Engility Corporation and Ramamohana Gubbala from Raytheon Technical Services. The authors would like to also acknowledge the NASA ATOL team for support in conducting the experiments. This research was supported by the National Aeronautics and Space Administration.

\section{Email Addresses}

Zahra Khan, zahra.khan@engilitycorp.com

Husni Idris, husni.idris@engilitycorp.com
Maria Consiglio, maria.c.consiglio@nasa.gov

David Wing, david.wing@nasa.gov

30th Digital Avionics Systems Conference

October 16-20, 2011 